\section{Choice-making and choose-ables}

Making decision agents more choosy

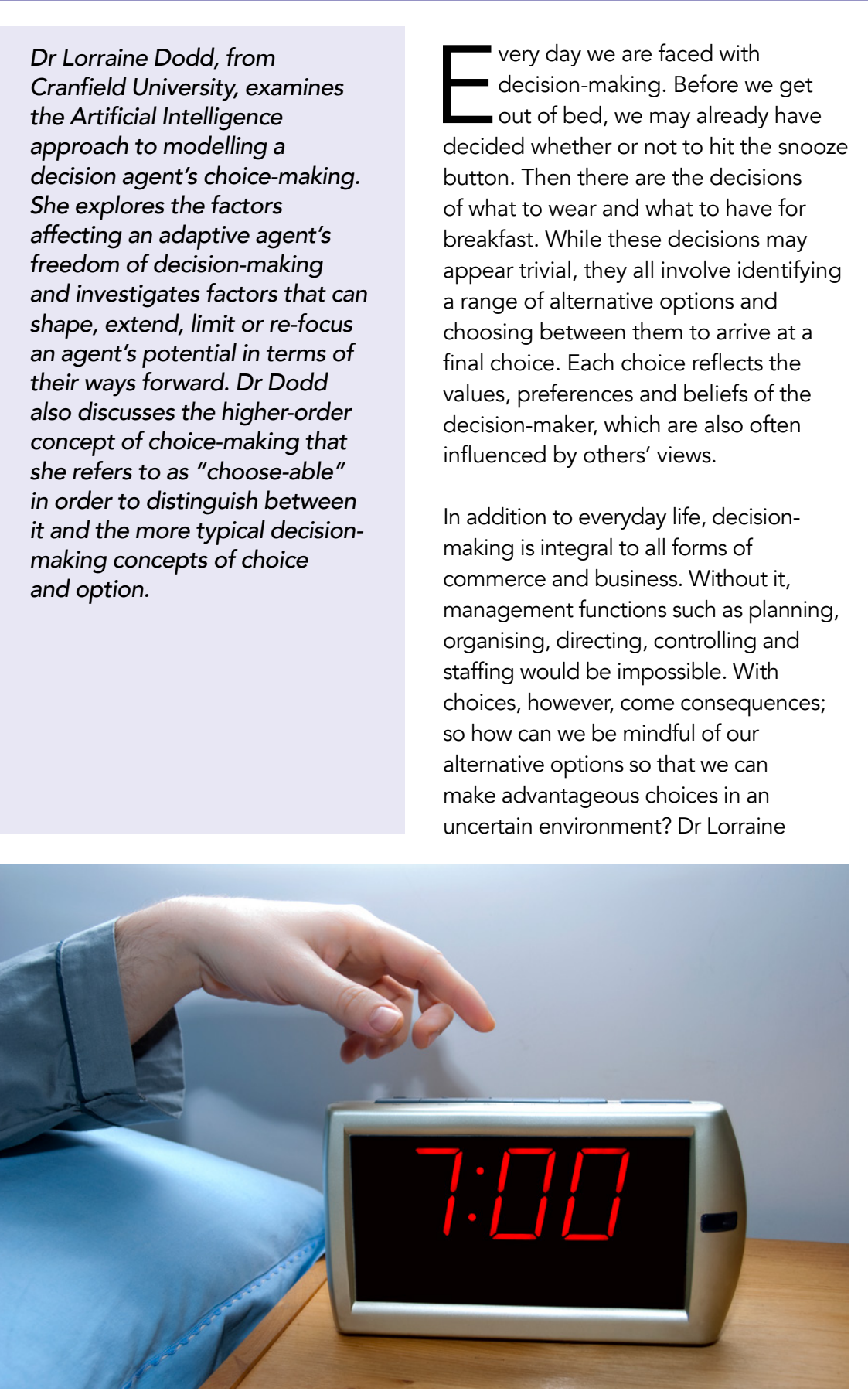

Dodd, Research Director in Applied Systems Studies at Cranfield University, Dr Dodd's research has inolved Dr Dodds research has involved studying This has established her as a highly respected international contributor to militany Command, Control, Intelligence and Information analysis.

The study of any decision-making process involves a combination of mathematics, statistics, psychology and philosophy. Dr Dodd's current research examines how artificial intelligence (Al) and mathematical algorithms model choice-making. She also explores the factors affecting an agent's freedom of decision-making, with particular interest in agents that have the ability to adapt. The mathematical basis that underlies these adaptive agents is fundamental to choice and as such can span from agenthrough to people, organisations and socio-political institutions.

\section{HIGHER-ORDER CHOICE-MAKING} Dr Dodd explains that fundamental to this research is the question: "what agent's potential in terms of their ways forward and felt degrees of freedom of choice?" She refers to this higher-order concept of choice-making in terms of what is "choose-able" so as to establish the distinction between a choose-able, which is subjective, and the more typical decision-makng concepts of choice and option, which tend to be objective.

CHOOSE-ABLES

Before they can choose, an agent has to assemble a list of things to choose

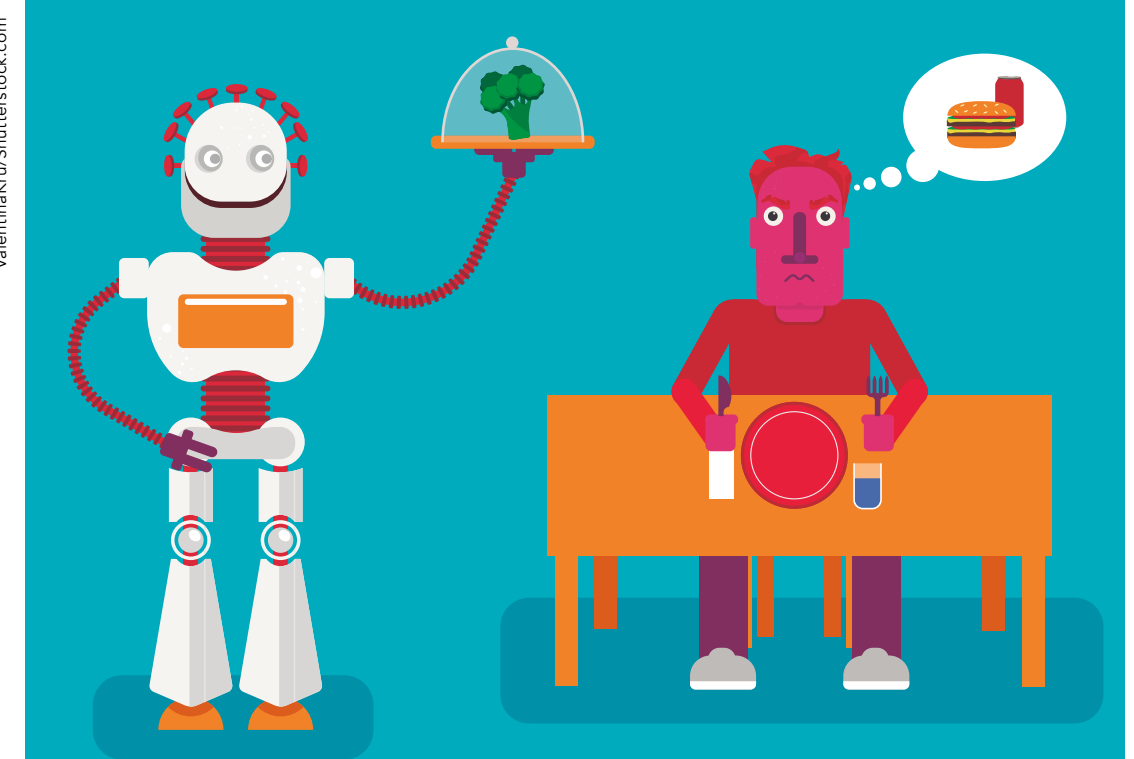

Sometimes only one choose-able is available to an agent.

What might shape, extend, limit or re-focus an agent's potential in terms of their ways forward?

from. This forms the agent's potential in rules and laws and feel that they have to terms of their scope of choose-ables and abide by them. The funnel narrows as the comprises all the possible ways forward agent hones in on the choose-ables that that they can imagine, deem acceptable they believe to be possible in their quest (e.g., within their moral code), consider open to them, feel obliged to consider, hink they are competent to consider and believe they are barred from considering (e.g., social taboos). Choose-ables tend to be influenced by the decision agent's circumstances and their relational conditioning. chequentive

Dr Dodd examines what can intrinsically or extrinsically affect an agent's degree of decision freedom in order to understand the conditions that shape the choose-ables that can lead to decisive consequences

\section{FUNNELLING CONSTRUCT} es, going from the imagined to those deemed possible, is depicted using a triangular funnelling construct. It shows the choose-ables in relation to the agent's capabilities and structured by both their motivation (i.e., highly motivated agents tending choose and and the der scope of hey accord with any associted which

\section{THEORY OF CHOIC}

Previously, Dr Dodd's research explored how choose-ables were likely to sculp how an agent might sense, feel, int act. She highlights how these actions of to the agent when they are faced with con have to frame the situation according to that particular way forward to provide the reassurance of having a solution. Dr Dodd uses the example of wester governments with their constraint of accountability and legal scrutiny, limiting them to just one choose-able, such. as imposing sanctions. Focussing on sanctions can restrict how they see and comprehend the situation. This suggests that the influences can flow both ways between understanding the situation and the choose-ables that are on offer.

\section{SOCIAL INFLUENCES}

Dr Dodd describes how an agent who is open to working with others and taking will have choose ables that include the others' options. This agent will also be disposed to finding compromises that are acceptable for everyone concerned, rather than finding the 'best' solution in terms of their own preference.

So, this research brings together Culture Theory and the mathematics of Catastrophe Theory to propose novel ways to analyse the effects of relational contexts on an agent's choose-ables.

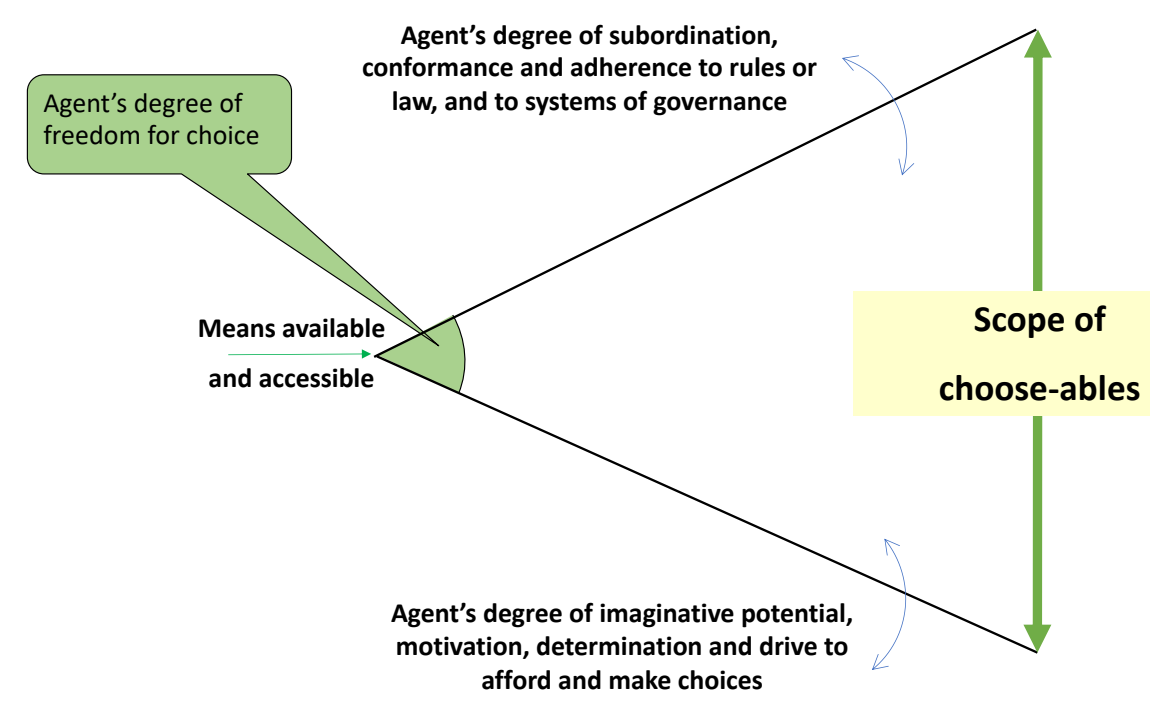


characterise an agent's choice-making in different relational contexts. For state, they will tend to advocate group norms and defined structure, group militany command and control culture. They will aim for a balanced resolution that takes other agents' perspectives into account. Conversely, if the agent is in an individualist state, they will tend towards their personal preference with little restriction from formal structures such as rules or ethics. Their choice will be steered by what they accept as norma for example, making profit.

The complex relational field that helps to distinguish between the four states also influences where an agent's sociocultural relationships might be either helping them to stay (i.e., attraction) negative e-motion). The circumstances in which agents happen to find

themselves can impact on the degree

thenselns any and heteronomy that they

will be able to accept.

\section{CATASTROPHE THEORY}

The application of Catastrophe Theory facilitates mathematical rigour in the modelling. Four shaping coefficients, a, $b, c$ and d, are associated with differen relational conditions and the ensuing

choose-ables.

The four shaping coefficients are:

a. The combination of 'normal' factors used to monitor and maintain an sense of 'OK-ness' can then focus choose ables down to adptive thresholds that are known to provide success, stability and survival.

b. Separating 'confusion' factors that represent the degree of uncertainty experienced due to a situation having two or more conflicting perspectives. Belief 'bias' factors used to comprehend biases and differences in projected outcomes as well as beliefs in their own and other agents' notions of possible outcomes

d. Assessment 'value factors to evaluate and resolve the agent's own and other agents' preferences and perspectives.

Dr Dodd notes that these four

coefficients can change over time as

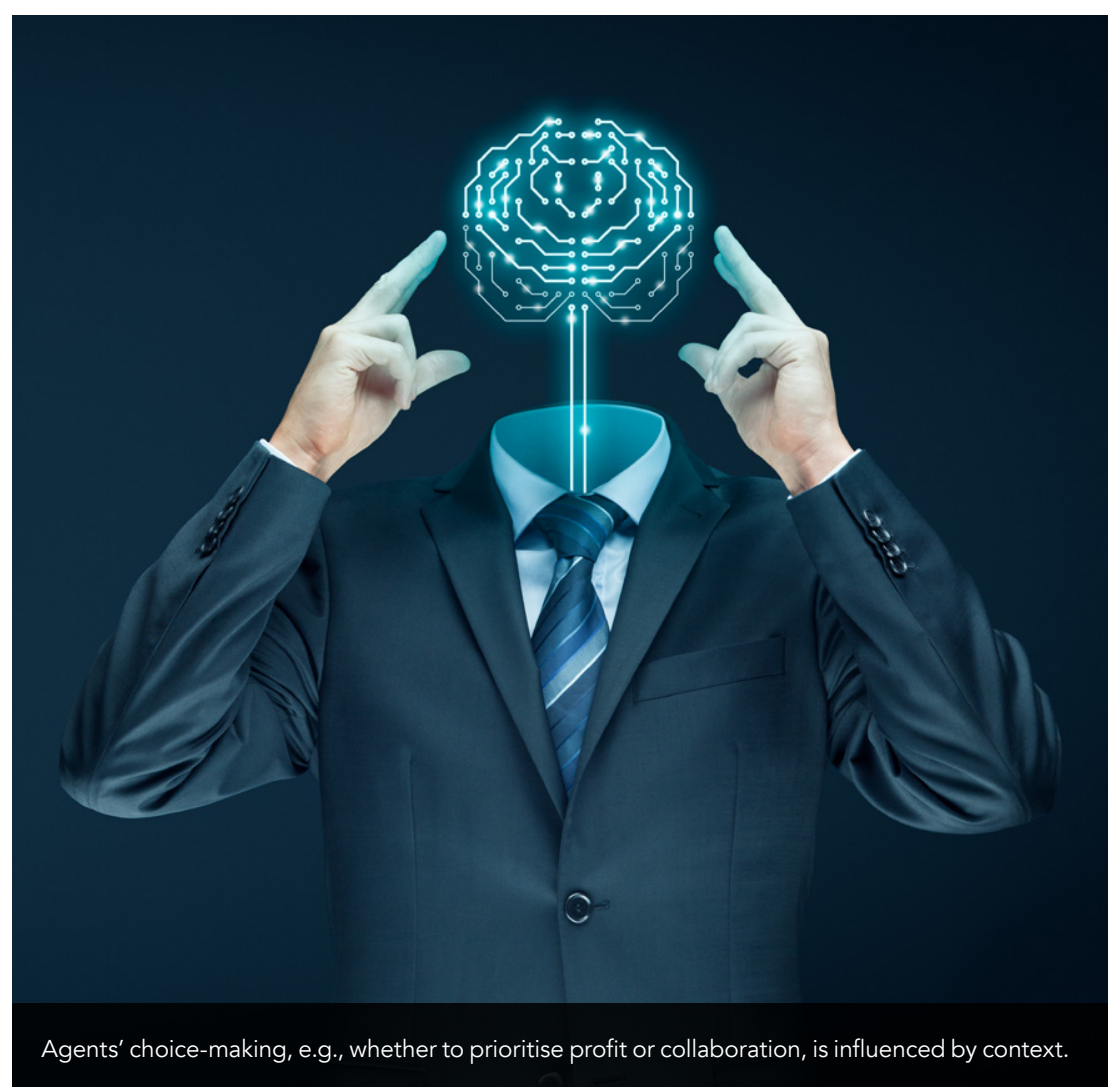

The influences can flow both ways between understanding the situation and the choose-ables that are on offer.

the relational circumstances change. Their relevance can also increase or Their relevance can also increase or
decrease when functional needs or living hitherto have tended to remain
hidden and tacit". This descriptive conditions change. power provides a rich language that pow ber provides a rich language that
can understand where an adaptive agent might be in terms of their choose-ables, as well as offering possible explanations as to why

The notion of a choose-able is very powerful. Often, we can only make inferences regarding the nature of choose-ables after a choice has been made and the ensuing actions observed. Dr Dodd's research provides insights into how the subjective concepts of subordination, rules, norms, taboos, beliefs, myths, biases, preferences and focus of attention can affect and shape an agent's choose-ables. These constructs can uncover the underlying assumptions concerning an agent's rationale and show how they arrived at a dilemma or chose to carry out a to Decision Theory and Game Theory.

\section{Behind the Research}

\section{Dr Lorraine Dodd}

E: L.dodd@cranfield.ac.uk T: +44 (0) $7875734228 \quad$ W: https:///www.cranfield.ac.uk/people/
lorraine-dodd-279815 W: http//bioss.com/about/people-and-partners/lorraine-dodd/

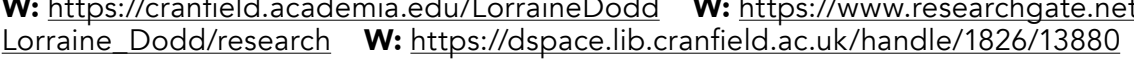

Research Objectives

Dr Dodd's work examines how Al and algorithms approach choice-making and
freedom of decision making.

\section{Detail}

Cranfield University Defence and Security School SNG 8 L

modelling around studies psychological mathematical people arond choice-making; particularly, when tusion and complexity. She has research expertise in artificial neural networks, human-machine learning and agent-based modelling; currently engaged intelligence agents to support critical decisions.

Funding

Collaborators

Thanks to great and kind colleagues in Cranfield colleagues at QinetiQ, in particular Dr Dave Marsay, Paddy Turner and Patrick Beautement, for many helpful, adaptation, open-eyes, open-minds and choice-making Sincere thanks to previous co-authors: Professor Jim Q Smith, Head of Mathematics and Statistics at Warwick Principal Analysts at OinetiQ; and, Professor Jim Mormer at Dstl with Graham Mathieson. Finally, Professor Gillia introducing me to the original l'ch, in particular for economist George LS Shackle, and mostly for everything

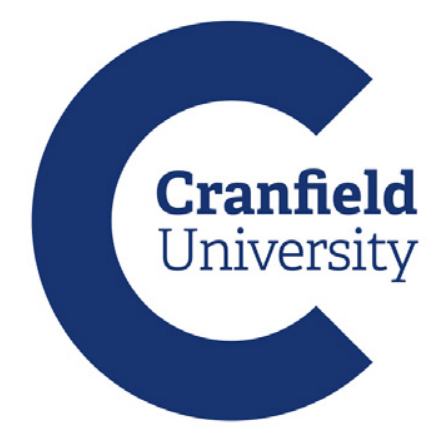

\section{References}

Dodd, L. (2019), Choice-making and choose-ables: making decision agents more human and choosy. Euro Journa org/10.1007/s40070-018-0092.-5

Dodd, L. (2019). Techne and techniques for engaging in a socially complex world. Journ
Research Society, $70(9), 1399-1409$ Dodd, L. and Smith, J. O (2013). Devolving command decisions in complex operations. Journal of the
Operational Research Society, 64 (1), 17-33. Dodd, L. and Markham, G. (2013). Orders of C2 agility and
implications for information and decision-making. Internation Command and Control Research Technology Symposium Institute of Defense Analysis, Virginia. CCRP Publications. Available at: https://apps.dtic.mil/dtic/tr/fulltext/u2/a587016,
pdffAccessed 10th October 2019] Dodd, L. (2011). A Theory of Choices: melding black swans, Conference on Complexity Science, June 2011, Boston, USA. Dodd, L. and Alston, A.J. (2009). Complex Adaptive and 'Inquiring' Systems Theory for Contemporary Military insurgency, Vienna. Available at: http://www.ismor. com/cornwallis/workshop_2009.shtml [Accessed 10th Dodd, L., Moffat, J., Smith, J.Q. and Mathieson, G. (2004).
Command decision-making with conflicting objectives: an exploration of some experimental data. ISMOR 2004. Available at: http:///ismor.cds.cranfield.ac.uk/21st-
symposium-2004 [Accessed 10th October 2019] Shackle, G. L. S. (1976) Time and Choice. Keynes Lecture in
Economics, Proceedings of the British Academy, 62, 309-329.

\section{Personal Response}

\section{What initially prompted your research into
choice-making and choose-ables?} II Being with people and also observing people as they
are coming to critical decisions, when analysis along the
tradititional lines of decision theory did not help to explain why certain choices had been made. I was introduced to two important areas of work: Drama Theory by Prof Jim
Bryant and Peter Bennett (developed by Nigel Howard Bryant and Peter Bennett (developed by Nige Howard
for military commanders as Confrontation Analysis); George L. S. Shackle's Subjective Choice Theory (i.e., the concept of a choose-able) by Prof Gillian Stamp. My
previous studies in Pure Mathematics at Warwick with prof Christopher Zeeman had left an indelible fascination Douglas and Thomson work on Culture Theory provided the way forward to develop a formal structural logic for
understanding choice-making. 